\title{
Importations et remaniements de thanatofaunes dans les sables de la plate-forme profonde des approches occidentales de la Manche
}

\author{
Jean-Yves REYNAUD* a, Agnès LAURIAT-RAGE ${ }^{\text {b }}$, Bernadette TESSIER a , Didier NERAUDEAU ${ }^{\text {c, }}$ \\ Éric BRACCINI ${ }^{\text {d }}$, René-Pierre CARRIOL ${ }^{\text {b }}$, Martine CLET-PELLERIN ${ }^{\mathrm{e}}$, Michel MOULLADE ? \\ Gilles LERICOLAIS ${ }^{\mathrm{g}}$ \\ ${ }^{a}$ Laboratoire de sédimentologie et géodynamique, université des sciences et techniques de Lille, \\ 59655 Villeneuve d'Ascq cedex, France \\ * reynaud@mnhn.fr \\ ${ }^{b}$ Laboratoire de paléontologie, Muséum national d'histoire naturelle, 8, rue Buffon, 75005 Paris, France \\ ${ }^{\mathrm{C}}$ Géosciences Rennes, université de Rennes-1, campus de Beaulieu, 35042 Rennes cedex, France \\ ${ }^{\mathrm{d}}$ Elf exploration-production, CSTJF - Remplissages sédimentaires - L1, 1092, avenue Larribau, 64018 Pau, France \\ ${ }^{\mathrm{e}}$ Centre de géomorphologie, université de Caen, rue des tilleuls, 14000 Caen, France \\ ${ }^{\mathrm{f}}$ Laboratoire de micropaléontologie et géologie marines, université de Nice-Sophia-Antipolis, Parc Valrose, \\ 06108 Nice cedex 2, France \\ ${ }^{g}$ Département géosciences marines, Institut français de recherche pour l'exploitation de la mer (Ifremer), \\ BP 70, 29280 Plouzané cedex, France
}

(Reçu le 5 mai 1998, révisé le 7 janvier 1999, accepté le 12 janvier 999)

\begin{abstract}
Introduction and reworking of thanatofaunas in deep shelf sediments of the Western Channel Approaches. Macro- and micro-faunas are reported from six gravity cores collected at a mean depth of $150 \mathrm{~m}$ in the surficial deposits of the Kaiser sand bank in the Southern Western Channel Approaches. These are bivalves (mainly), gastropods, echinids, crustaceans and foraminifers. These fauna present a very rich association of numerous, well preserved species from various ecological settings. Such an association is commonly reported from the continental shelf of the last transgressive cycle. In addition, the sediment comprises two imported faunal associations. The first one corresponds to thanatocaenoses of Pliocene/lowermost Pleistocene and Weichselian age, reworked respectively from the underlying celtic incised valley fills and the Celtic Sand Banks. The absence of Quaternary fauna prior to the Weichselian suggests that the introduction of Pliocene sources occurred during the last climatic cycle. The second stage of importation took place after the last transgression in the Western Channel, and corresponds to the enrichment of the sediment in coastal fauna derived from western Brittany by the the predominant ebb tidal current. The variations of taxonomic diversity from core to core suggest a sediment transport around the bank that matches the one deduced from the study of tidal bedforms. At the present day, the wave action is highlighted by accumulation of species of the same shape, size and density inside storm beds. However, the survival within these beds of very small, juvenile shells indicate that each storm reworking was short in duration. (C) Elsevier, Paris / Ifremer / CNRS / IRD
\end{abstract}

\section{continental shelf / thanatofauna / sea level / reworking / transport}

Résumé - Cette étude concerne les thanatofaunes de six carottes gravitaires prélevées sur un banc de la plate-forme profonde des approches occidentales de la Manche. Les associations de faune sont riches en individus entiers ou en fragments bien conservés et montrent une forte diversité écologique, qui reflète l'enregistrement du dernier cycle transgressif sur la plate-forme continentale. Elles montrent en outre deux étapes d'importation faunique. La première correspond à l'introduction dans le sédiment superficiel de deux thanatocếnoses d'âges Pliocène-Pléistocène basal et Weichsélien, qui pro- 
viendraient respectivement du remaniement des vallées incisées et des bancs de la mer Celtique. L'absence d'espèces quaternaires antérieures au Weichsélien suggère que l'introduction des thanatofaunes pliocènes se soit produite lors du dernier cycle climatique. La deuxième phase d'importation se fait à la suite de la dernière transgression de la Manche occidentale et correspond à l'enrichissement du sédiment en espèces originaires du littoral ouest-armoricain sous l'effet du jusant dominant. Les variations de diversité taxonomique d'une carotte à l'autre et l'accumulation de spécimens de même mobilité dans des passées représentant des dépôts de tempêtes suggèrent une dynamique sédimentaire en accord avec le modèle issu de l'analyse des sonogrammes. (C) Elsevier, Paris / Ifremer / CNRS / IRD

plateau continental / thanatofaune / niveau de la mer / remaniement / transport

\section{INTRODUCTION}

Le système des sédiments superficiels de la plate-forme profonde de la façade atlantique française pose un problème d'ordre génétique depuis la découverte par Bourcart [7] de sa non-continuité avec le système terrigène côtier. Dans la Manche, cette non-continuité est caractérisée par une zone où prédominent des sédiments sabloargileux mal triés [10,27], entre les systèmes bio-lithoclastiques de la Manche occidentale (en amont) et de la mer Celtique méridionale (en aval). Cette zone se situe vers $120 \mathrm{~m}$ de profondeur dans le secteur des grands bancs de la mer Celtique (figure I), à proximité de la ligne de régression maximale du Quaternaire [36]. Une différenciation de ces ensembles sédimentaires superficiels par les variations du niveau marin peut être envisagée avec, de part et d'autre de la ligne de bas niveau marin : (1) le domaine de la Manche, qui était exondé en période glaciaire, comme en témoignent des dépôts périglaciaires autochtones $[27,38,43] ;(2)$ un domaine de plate-forme distale (couvrant en particulier les grands bancs de la mer Celtique méridionale) qui semble être resté marin tout au long du Quaternaire [48], mais soumis en période de bas niveau à l'influence proximale du «Fleuve Manche » $[22,42]$ et à celle des débâcles tardiglaciaires [48].

En conséquence, alors que la fraction organogène marine caractéristique de la Manche occidentale est essentiellement flandrienne et holocène [38], celle de la mer Celtique méridionale (plate-forme profonde des approches occidentales de la Manche) est principalement constituée par un mélange de faunes enrichi continûment au cours du Quaternaire. Cela est suggéré, dans cette fraction, par une abondante malacofaune avec des spécimens de grande taille montrant des traces d'usure et d'oxydation non observées en Manche, ainsi que par des tubes de vers fossiles (Ditrupa arietina) [10]. La formation superficielle d'épaisseur métrique contenant ces faunes repose sur un niveau décimétrique de graviers à blocs [48], inter- prété comme un reliquat du vannage sous-marin associé à la dernière débâcle tardiglaciaire vers 13-14 Ka. Dans les sables superficiels de la mer Celtique méridionale, la présence de taxons antérieurs à la dernière glaciation suggère un remaniement postglaciaire de stocks sableux, localisés sous la discontinuité graveleuse, que pourraient représenter les grands bancs ou le comblement des vallées incisées de la mer Celtique $[9,51]$ (figure 1).

De nouveaux éléments, fondés principalement sur l'analyse taxonomique, permettent de préciser, d'une part, les sources sédimentaires et l'âge du remaniement dans les dépôts superficiels de la plate-forme profonde des approches occidentales de la Manche et, d'autre part, les directions de transport sédimentaire à long terme.

\section{MATÉRIEL ET MÉTHODES}

Les résultats présentés concernent les dépôts superficiels d'un banc sableux, le banc du Kaiser, couvrant une superficie de $300 \mathrm{~km}^{2}$ environ (figure 1). Ce banc est l'objet d'une étude multidisciplinaire sur les bancs tidaux et les formations plio-quaternaires de la Manche [51]. Des prélèvements fauniques ont été réalisés sur six carottes Kullenberg d'un diamètre de $14 \mathrm{~cm}$ et d'une longueur variant entre 0,6 et $2 \mathrm{~m}$, réalisées durant la campagne Sédimanche 2 (tableau $I$ ).

Les tubes utilisés ne mesurant que $3 \mathrm{~m}$, nous avons choisi la position des carottes d'après des enregistrements de sondeur multifaisceaux (Simrad EM1000) et de sismique à très haute résolution (sparker SIG) de manière à éviter les plus grands corps dunaires présents à la surface du banc ( $t a b l e a u ~ I$ ). En effet, la dynamique active de ces derniers suggère un vannage des sables qu'ils contiennent, ce qui est confirmé par l'absence de grosses coquilles dans les carottes prélevées en domaine dunaire ( $\mathrm{KSO} 3$ et KS07 ; tableau I). Le sédiment moins mobile entre les dunes ou les rubans sableux, plus représentatif de l'ensemble du système superficiel [51], a été carotté. La 


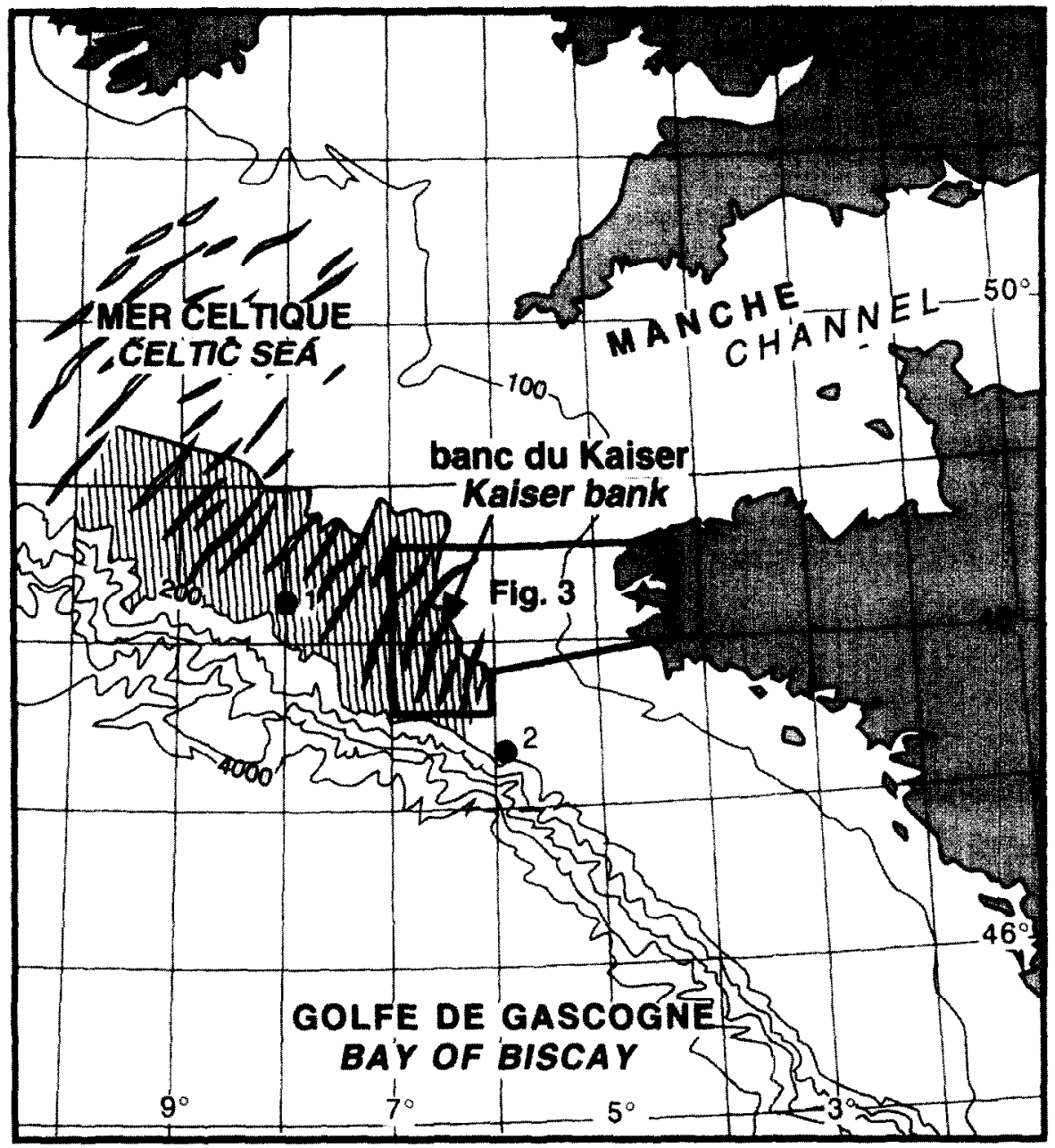

Figure 1. Cadre géographique et hydrodynamique du secteur d'étude. Les grands bancs de la mer Celtique sont représentés en grisé. Le secteur hachuré correspond au domaine des vallées incisées de la plate-forme profonde. Les pastilles noires figurent les points de mesure du courant de fond ; $1:$ site du forage Krogen ; 2 : station $n^{\circ} 1$ de Cavanié et Hyacinthe [15].

Figure 1. Location of the study area. The Celtic Banks are reported in shadow. Hatched area shows the extent of incised valleys on the outer shelf. Black spots show locations of sea-floor current measurements; 1: Krogen borehole; 2: station 1 in Cavanié and Hyacinthe [15].

Tableau I. Caractéristiques des sites carottés.

Table I. Typical features of cored targets.

\begin{tabular}{|c|c|c|c|c|c|}
\hline Carotte & $\begin{array}{l}\text { Site } \\
\text { (/courant dominant) }\end{array}$ & Profondeur & $\begin{array}{l}\text { Morphologie } \\
\text { du site }\end{array}$ & $\begin{array}{l}\text { Dynamique } \\
\text { prédominante }\end{array}$ & $\begin{array}{l}\text { Mobilité sédimentaire } \\
\text { (/rétrodiffusivité acoustique) }\end{array}$ \\
\hline KSO3 & queue de banc & $147 \mathrm{~m}$ & dunaire relique & tidale intermittente & moyenne \\
\hline KS04 & crête de banc & $131 \mathrm{~m}$ & zone plane & houle forte & faible \\
\hline KS05 & pied de banc & $157 \mathrm{~m}$ & interdunaire 3D actif & tidale forte & très forte \\
\hline KS06 & pied de banc & $153 \mathrm{~m}$ & interdunaire $2 \mathrm{D}$ actif & tidale forte & forte \\
\hline KS07 & pied de banc & $157 \mathrm{~m}$ & interrubans actif & tidale forte & moyenne \\
\hline KS08 & tête de banc & $132 \mathrm{~m}$ & petites dunes & houle intermittente & faible \\
\hline
\end{tabular}


couche graveleuse décrite à la base de ce système par Pantin et Evans [48] n'a pas été mise en évidence et la relative homogénéité lithologique des carottes suggère que celles-ci n'ont pas atteint la formation sous-jacente des bancs de la mer Celtique.

Des prélèvements systématiques de $20 \mathrm{~g}$, ponctuels ou en continu, ont été effectués sur les carottes (figure 2). Une analyse palynologique a été réalisée sur la fraction comprise entre 10 et $125 \mu \mathrm{m}$ (M. Clet-Pellerin). Dans la fraction supérieure à $125 \mu \mathrm{m}$, les faunes ont été déterminées pour les bivalves (A. Lauriat-Rage), les échinides (D. Néraudeau), les gastéropodes (D. Néraudeau), les cirripèdes (R.-P. Carriol) et les ostracodes (E. Braccini). L'étude des foraminifères (M. Moullade et A. El Hakour) a été focalisée sur des prélèvements à la base des carottes.

\section{RÉSULTATS}

\subsection{Contexte sédimentaire}

Les sables carottés sont majoritairement bioclastiques ( 55 à $75 \%$ de $\mathrm{CaCO}_{3}$ ), de granulométrie moyenne (médiane de $0,3 \mathrm{~mm}$ pour $\mathrm{KSO} 3$ et $\mathrm{KSO4}$ ) à grossière (médiane de $1 \mathrm{~mm}$ pour $\mathrm{KS} 05$ à $\mathrm{KS} 08$ ), de couleur beige clair à rousse, localement liés par une boue carbonatée au niveau de rares passées décimétriques (figure 2). Le sédiment présente une lithologie assez homogène, sans changement marqué entre la base et le sommet des carottes. La granularité du sédiment augmente du sud au nord du banc, en accord avec les résultats antérieurs [10]. La faible proportion de sédiments fins s'explique par leur vannage progressif, au cours de la transgression holocène, par les courants de marée et de houle, conformément au modèle de McCave [44]. Sur la plate-forme distale, cet appauvrissement en sédiments fins n'a pas été compensé par les apports terrigènes, principalement piégés en amont dans les prismes littoraux de la Manche [38]. La fraction fine terrigène qui pourrait atteindre le domaine des approches occidentales de la Manche demeure actuellement en transit jusqu'à l'océan [1].

De même qu'en Manche, les courants de marée contrôlent le transport sédimentaire en mer Celtique et sur la plate-forme profonde [27]. Dans le secteur d'étude, ils peuvent atteindre $0,35 \mathrm{à} 0,7 \mathrm{~m} \cdot \mathrm{s}^{-1}$ près du fond $[12,28$, 51]. Les courants les plus forts ont été mesurés au voisinage de la rupture de pente de la plate-forme, et résulteraient en partie d'une onde de marée interne en période estivale [28]. Les courants peuvent déplacer des sables bioclastiques grossiers jusqu'au voisinage de la rupture de pente du plateau continental $[29,28]$. La prédominance du jusant sur toute la plate-forme externe explique les transports sédimentaires vers le sud-ouest, d'après la migration de dunes observée sur les enregistrements bathymétriques $[9,13]$, de sonar à balayage latéral [18, 19], les photographies sous-marines [27] et les données de sondeur multifaisceaux [8].

Généralement, les carottes présentent à leur sommet un sédiment plus fin, de couleur claire et pauvre en malacofaune (figure 2), dont l'épaisseur, à profondeur égale, semble contrôlée par la topographie (tableau I) : il atteint $50 \mathrm{~cm}$ dans les espaces interdunaires de pied de banc (KS06) et est absent des zones non dunaires caractéristiques du sommet du banc (KS04). Ce dépôt est interprété comme la resédimentation des particules fines mises en suspension par les tempêtes [51]. Dans le secteur d'étude, la houle induit des courants alternatifs sur le fond, dont la vitesse instantanée atteindrait $35 \mathrm{~cm} \cdot \mathrm{s}^{-1}$ pendant $5 \mathrm{j}$ par an à $100 \mathrm{~m}$ de profondeur [14]. À l'intérieur du corps principal de certaines carottes, des passées centimétriques de graviers ou de sables à matrice plus fine soulignent localement un discret litage qui pourrait être associé à d'autres séquences de non-dépôt/vannage/resédimentation (figure 2). La houle, si elle n'est pas directement responsable de la remobilisation du sédiment, agirait efficacement en couplage avec les courants de marée [53]. De grandes rides symétriques (gravel ripples), présentes jusque dans les secteurs les plus profonds de la mer Celtique, témoignent de l'importance des mouvements sédimentaires induits par la houle $[16,51]$.

\subsection{Faune des thanatocénoses}

Dans la fraction inférieure à $125 \mu \mathrm{m}$, l'analyse des sédiments a permis d'identifier quelques dinoflagellés mais aucun pollen, ce qui suggère soit l'absence de dépôt, soit un remaniement intense des sables ayant conduit à l'abrasion et à l'oxydation de la matière organique molle. Dans la fraction supérieure à $125 \mu \mathrm{m}$, l'étude a été plus particulièrement développée sur les bivalves, qui représentent la plus grande partie de la macrofaune. Parmi les autres groupes déterminés, on note, par ordre d'abondance décroissante, les cirripèdes, les gastéropodes, et les échinides. La microfaune présente des ostracodes et des foraminiferes, ces derniers n'ayant été étudiés qu'à la base des carottes. Les résultats de l'analyse faunique (figure 2) 
conduisent aux interprétations suivantes en termes d'âge et de milieu de vie.

\subsubsection{Bivalves}

L'étude de cette faune fait principalement référence aux travaux de Tebble [56]. La composition faunique des bivalves varie peu d'une carotte à l'autre. Certaines espèces sont observées dans la plupart des niveaux des six carottes et bien représentées en nombre de spécimens (ex. Chlamys opercularis, Gouldia minima et Timoclea ovata). Cependant, certaines espèces ne sont présentes que dans une seule carotte, avec un spécimen seulement : Bathyarca pectunculoides et Palliolum similis dans KS04, Striarca lactea dans KS05, Plagiocardum papillosum dans KSO8 (figure 2). Contrairement aux gastéropodes, la variabilité des couleurs et du degré d'usure des spécimens de bivalves rencontrés, ainsi que le mélange d'espèces épibenthiques et endobenthiques, à caractère infralittoral (Spisula ovalis, Venus casina, Glycymeris glycymeris) et circalittoral (Pododesmus squamulus, Timoclea ovata) constituent des arguments en faveur d'un mélange important d'espèces d'âges géologiques et de biotopes différents, en accord avec les hypothèses formulées pour une partie des crustacés et des foraminifères.

En raison de la petite taille de la plupart des spécimens observés et de leur état souvent très fragmenté, la déter- mination spécifique des bivalves n'a pas toujours été possible. Cette thanatofaune comprend 40 taxons dont l'identification se situe pour certains au niveau de la famille (seulement 23 espèces déterminées). Elle est donc taxonomiquement plus pauvre qu'il n'y paraît, car les taxons supraspécitiques se rattachent presque toujours à une espèce déjà citée dans la même famille ou le même genre. Cette oligospécificité tient au fait que, par rapport aux autres groupes, davantage d'espèces de bivalves ont leur biotope plutôt dans le domaine infralittoral supérieur (généralement moins de $40 \mathrm{~m}$ de profondeur), moins diversifié du point de vue faunique que le domaine circalittoral-infralittoral inférieur des côtes bretonnes ou d'Aquitaine [17].

Les espèces qui conservent une très petite taille à l'âge adulte sont rares (Limatula subauriculata, Goodallia triangularis). La grande majorité des coquilles est composée de stades jeunes d'espèces de taille petite à moyenne (Pododesmus squamulus, Spisula ovalis, Gouldia minima, Timoclea ovata) et de formes très juvéniles (prodissoconque parfois conservée). De cette population, Pododesmus squamulus constitue l'espèce prédominante. Une telle ségrégation des individus les plus mobiles pourrait s'opérer à la faveur des remaniements périodiques du sédiment mis en évidence dans la lithologie des carottes. Par rapport à la grande richesse en individus ( 15 à 22 spécimens par carotte), l'oligospécificité des associations

Figure 2. Description lithologique et contenu thanatofaunique de quelques groupes étudiés dans les carottes Sédimanche 2 (implantation voir figure 3). Lithologie: STG : sable très grossier (médiane 1,2-2,5 mm) ; SG : sable grossier (médiane $0,7-1,2 \mathrm{~mm}$ ) ; SM : sable moyen (médiane $0,34-0,7 \mathrm{~mm}$ ) ; SF : sable fin (médiane $0,16-0,34 \mathrm{~mm}$ ). Contenu faunique : Les barres noires signalent la présence d'un ou plusieurs taxons dans le ou les échantillons compris dans l'intervalle de profondeur correspondant. Les numéros renvoient aux taxons de la liste cidessous.

Figure 2. Lithology and faunal content of gravity core KS03 (cxcept Foraminifcra). Lithology: VCS: very coarse sand (mean 1.2-2.5 mm); CS: coarse sand (mean 0.7-1.2 mm); MS: medium sand (mean 0.34-0.7 mm); FS: fine sand (mean 0.16-0.34 mm). Faunal content: thick bars display the occurrence of one or several taxons in the sample(s) within the corresponding depth range. Numbers correspond to the following taxons:

Echinides (Echinids): 1: Cidaridae, 2 : Echinocyamus pusillus, $3:$ Echinocardium cordatum, $4:$ Spatangus purpureus, $5:$ Sphaerechinus granularis, 6 : oursin régulier indét. (non det. endocyclic sea-urchin), $7:$ oursin irrégulier indét. (non det. exocyclic sea-lurchin); Cirripèdes (Cirripedes) : $8:$ Scalpellum scalpellum, 9 : Verruca stroemia, $10:$ Chirona hameri, $11:$ Balanus crenatus, $12:$ Balanoidea indet. ; Ostracodes (Ostracods) : 13 : Aurilla convexa, $14:$ Eucythera cf. declinis, $15:$ Heterocuthereis cf. albomaculata, $16:$ Hirshmania tamarindus, $17:$ Loxocancha sp.; Gastéropodes (Gastropods) : 18 : Architectonica, $19:$ Balcis alba, $20:$ Balcis crosseana, $21:$ Bittium reticulatum, $22:$ Buccinum undatum, 23 : Calliostoma zizyphinium, 24 : Cantharus dorbignyi, 25 : Gibbula cineraria, $26:$ Gibbula tumida, $27:$ Nassarius reticulatus, 28 : Natica sp., 29 : Ocenebra sp., 30 : Scala mesogonia, 31 : Turritella sp.; Bivalves (Bivalvs) : $32:$ Anomia ephippium, $33:$ Anomia patelliformis, 34 : Anomia sp., 35 : Anomiidae indét., 36 : Barbatia cf. barbata, $37:$ Bathyarca pectunculoides, 38 : Cardiidae indét., $39:$ Chlamys opercularis, 40 : Chlamys islandica, 41 : Chlamys varia, 42 : Chlamys sp., 43 : Clausinella fasciata, $44:$ Glycymeris glycymeris, $45:$ Glycymeris sp., 46 : Goodallia triangularis, 47 ; Gouldia minima, 48 : Heterodonta indét., 49 : Hiùtella arctica, 50 : Hiatella rugosa, 51 : Hiatella sp., 52 : Limaria sp., 53 : Limatula subauriculata, 54 : Macoma sp., $55:$ Mactra sp., 56 : Mytilidae indét., $57:$ Nucula sp., $58:$ Ostrea edulis, 59 : Ostrea sp., 60 : Palliolum similis, 61 : Plagiocardium papillosum, 62 : Pododesmus squamulus, 63 : Spisula ovalis, 64 : Spisula sp., 65 : Spisula subtruncata, 66 : Striarca lactea, 67 : Tapetinae indét., 68 : Tellinidae indét., 69 : Timoclea ovata, $70:$ Veneridae indét., 71 : Venus casina. 
STG SG SM ST Echantillonnage Echinides/Cirripèdes Ostracodes/Gastéropodes Bivalves
VCS CS,MS, FS

KSO3
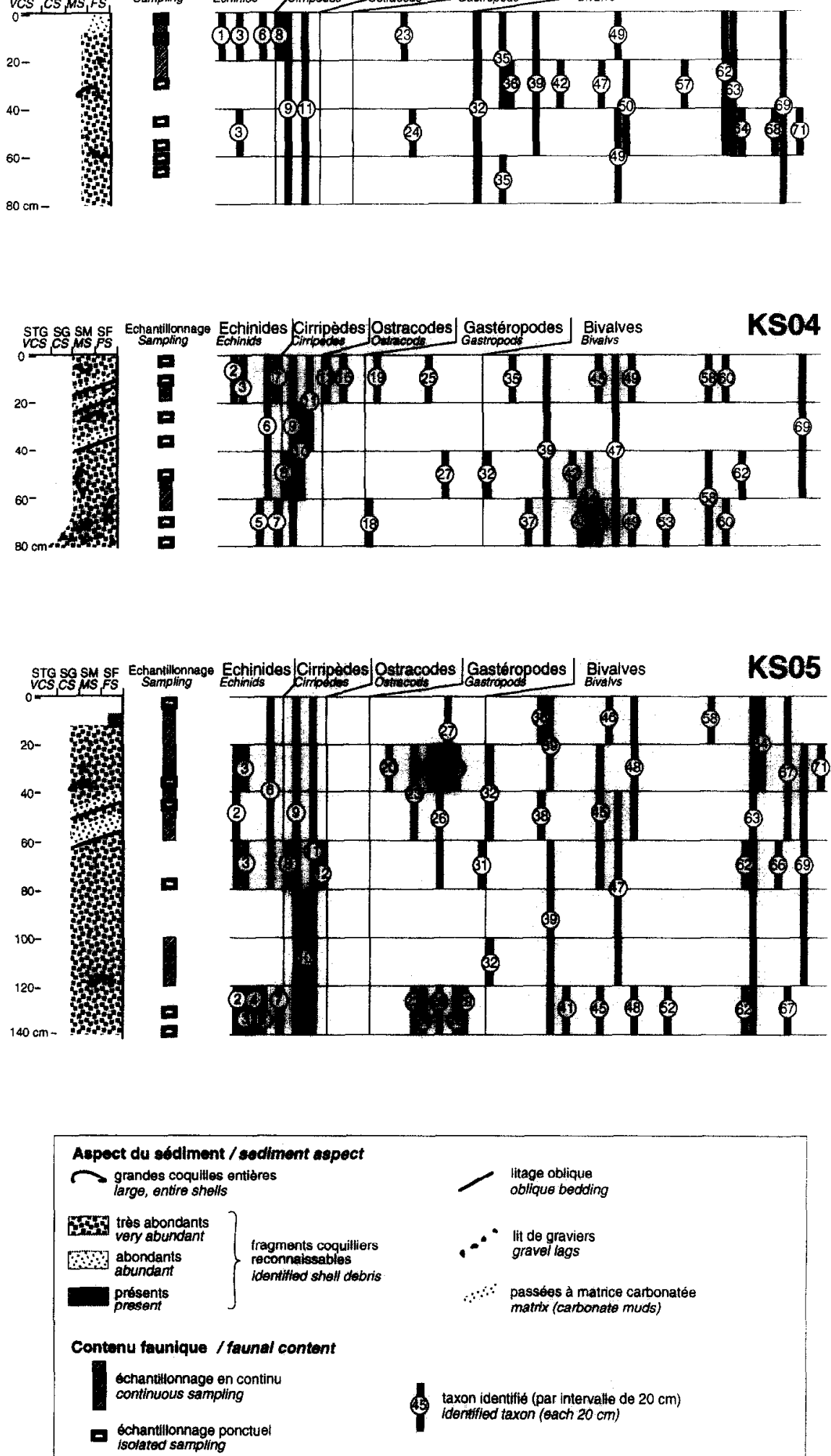

6 taxon identifie (par intervalle de $20 \mathrm{~cm}$ ) identified taxon (eact $20 \mathrm{~cm}$ ) 

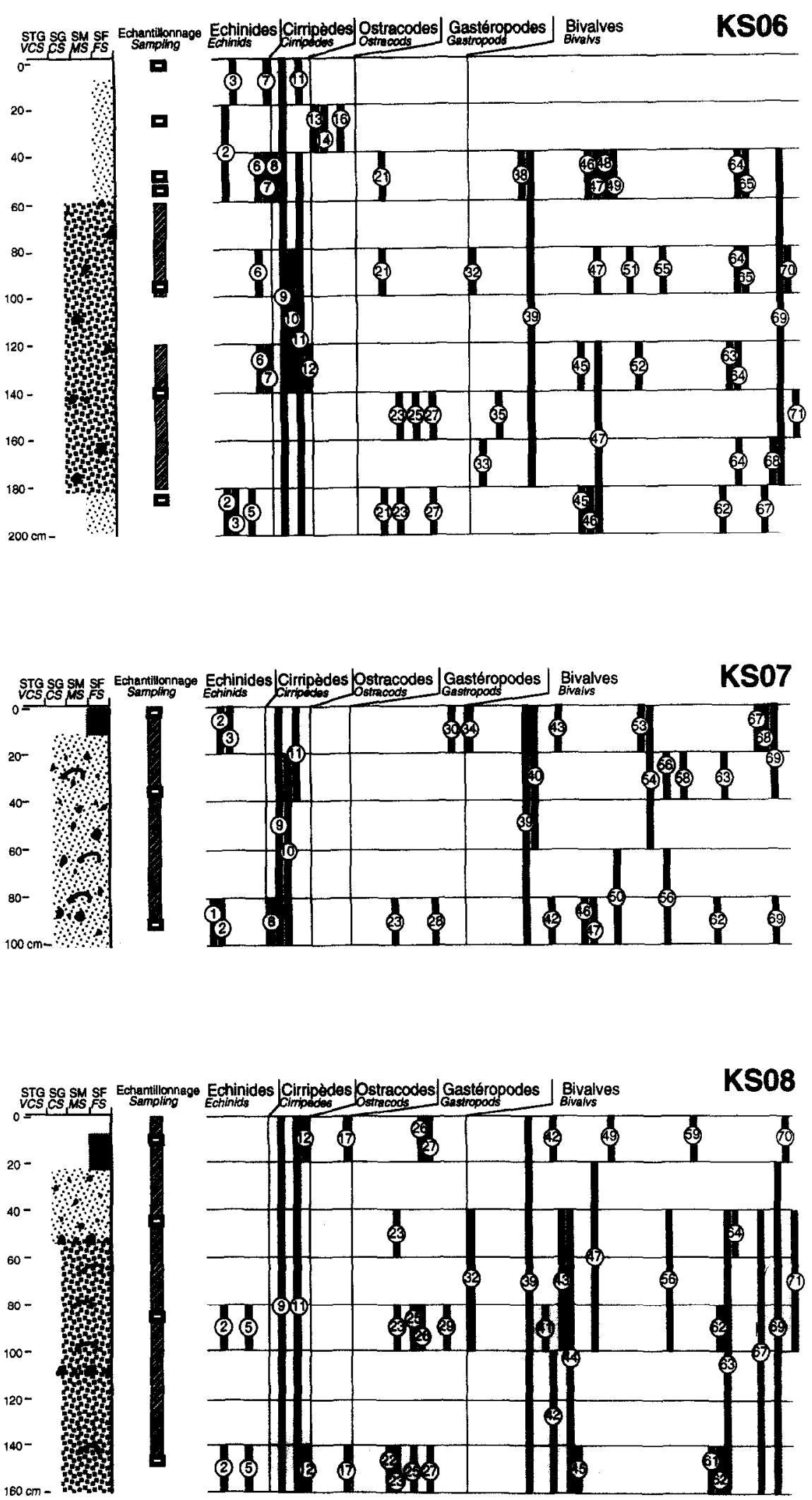
observées s'expliquerait par cette ségrégation fondée sur la forme, la taille et la densité des spécimens mobilisés.

Un autre argument en faveur du remaniement d'une fraction des bivalves observés est la présence très fréquente d'épizoaires des deux côtés des valves des plus grands spécimens : bryozoaires, serpules et foraminifères encroûtant les Chlamys et Glycymeris. Cela laisse supposer que l'on est en présence de coquilles ayant longuement séjourné sur le fond marin après la mort de leur occupant. La mobilité du sédiment est attestée par les transports déduits de l'analyse faunique. Outre les espèces côtières d'origine vraisemblablement bretonne, certaines espèces actuelles, de couleur jaunâtre, absentes aujourd'hui de la région du banc du Kaiscr, comme par excmple Chlamys islandica et Macoma sp. (KS07; figure 2), présentent un caractère arctico-boréal marqué $[23,24]$. Ces espèces, par leur couleur, leur degré d'usure, leur densité et leur taille pluricentimétrique, semblent appartenir à la même population que des espèces cosmopolites telles que Chlamys opercularis, Anomia ephippium, Hiatella rugosa [56]. Cela suggère qu'une partie importante du sédiment pourrait provenir du nord.

Ces espèces étant présentes au début du Tardiglaciaire dans la zone d'étude, on ne peut exclure qu'une partie d'entre elles soit héritée in situ de la dernière période froide, qui serait typée par des individus de couleur jaunâtre à fauve. Cette hypothèse est confortée par la présence dans KS07 (figure 2) de Chlamys islandica connue dans le Crag récent de l'East Anglia [62] - en association avec un foraminifère indicateur du même climat froid (Neogloboquadrina humerosa). Par ailleurs, Barbatia cf. barbata, mélangée dans KS03 à des cirripèdes et des foraminifères de couleur orangée à fauve, est un indicateur de climat méditerranéen, et ne vécut en Manche que jusqu'à la fin du Pliocène [39]. Les populations de couleurs jaunâtre et orangée à fauve pourraient donc résulter de la concentration de spécimens d'âges variés (Quaternaire et Pliocène) non détruits par l'abrasion marine.

\subsection{2. Échinides}

Ils correspondent à des espèces actuelles typiques d'une échinofaune d'oursins fouisseurs. Les associations en présence sont peu diversifiées. La totalité des espèces est commune sur la plate-forme proximale entre 20 et $40 \mathrm{~m}$ de fond, en particulier Echinocyamus pusillus, caractéristique des substrats graveleux $[49,58]$ et très répandu dans le maërl des côtes bretonnes [35], et Echinocardium cor- datum, qui vit dans les sables et sables vaseux infralittoraux, voire intertidaux, des côtes de la Manche et d'Aquitaine [35, 11, 41, 19]. Seul Echinocyamus pusillus peut vivre sur la plate-forme profonde. C'est probablement pourquoi il est ubiquiste dans les échantillons étudiés. De plus, les Echinocyamus pusillus observés sont blancs et non pas jaunâtres, comme souvent dans les zones vaseuses favorisant le piégeage de fer ferrique dans la microporosité structurale de leur test. Cette espèce peut donc être autochtone. Outre leur petite taille qui les rend moins sensibles à l'usure, cela expliquerait pourquoi les Echinocyamus pusillus sont plus souvent entiers. Une autre explication serait le transport de toute l'association infralittorale vers les zones plus profondes, avec «lavage " des Echinocyamus pusillus dans les eaux pauvres en sédiments argileux de la plate-forme celtique. Cette hypothèse est plausible, dans la mesure où la plupart des autres espèces d'oursins observées dans les carottes ont leur biotope plus en amont sur la plate-forme.

\subsubsection{Cirripèdes}

L'assemblage des quatre espèces identifiées n'est pas commun de nos jours en Manche, sauf dans des formations du Plio-Pléistocène. Actucllement, les bancs de Chiroma hameri les plus voisins de la zone d'étude sont en mer d'Irlande et mer du Nord au nord d'une ligne Galway-Liverpool $[18,54]$, ce qui n'exclut pas (1) soit de découvrir une colonie vivante plus méridionale, (2) soit de mettre en évidence une association en provenance du nord. Néanmoins, la couleur orangée de la plupart des pièces de Balanus crenatus contraste avec celle des Balanus crenatus vivants, d'un blanc éclatant. Cela est à rapprocher de la coloration des «sables roux » weichséliens de la plate-forme distale [7], qui serait due à une période de résidence dans la grande vasière sud-armoricaine [30]. Celle-ci n'ayant atteint la zone d'étude que lors des derniers épisodes glaciaires, il est probable que l'association de cirripèdes soit constituée de carapaces pré-holocènes autochtones remaniécs. Toutcfois, on ne note pas la présence de Balanus balanus, typique des associations d'âge Pleistocène dans cette zone.

\subsubsection{Ostracodes}

Les carottes contiennent très peu de carapaces d'Ostracodes. Les espèces représentées correspondent à une microfaune très commune sur le littoral atlantique et sont connues dès la base du Quaternaire [25]. Comme les carapaces sont abîmées, roulées, voire cassées, on en 
déduit qu'une fraction du sédiment est importée actuellement de secteurs plus internes de la plate-forme ou remaniée in situ à partir de matériel associé à des niveaux plus anciens. Les données ne permettent pas de trancher entre les deux hypothèses, toutes deux argumentées par les interprétations d'autres groupes fauniques.

\subsubsection{Gastéropodes}

Les associations de gastéropodes correspondent à des espèces actuelles dont les plus fréquentes, les gibbules, sont typiques des substrats rocheux ; d'autres formes, telles que Nassarius ou Turitella, sont généralement associées à des substrats sablo-vaseux, mais leurs fragments sont minoritaires dans les échantillons Sédimanche 2 $[6,49]$. Il s'agit donc d'un assemblage allochtone. Les couleurs et les ornementations initiales conservées ainsi que l'aspect très frais de certains spécimens (pas d'épifaune) suggèrent que (1) la durée du transport est courte et que (2) le dépôt et l'enfouissement des sables carottés est récent et rapide, probablement de l'ordre de quelques mois à peu d'années.

\subsubsection{Associations de foraminiferes \\ observées à la base des carottes}

Les foraminifères ont fait l'objet d'une étude focalisée sur les échantillons à la base des carottes (ogives Kullenberg). Les associations de foraminifères sont marquées par une forte à assez forte prédominance des formes benthiques par rapport aux formes planctoniques (10 à $25 \%$ ). Les populations benthiques sont surtout composées de Cibicididae, Nonionidae, rares Elphidiidae, Miliolidae. Les agglutinants (Textulariidae) sont également présents. Chez les planctoniques, les espèces dominantes sont surtout des formes non carénées telles que Globigerina bulloides, G. falconensis, Neogloboquadrina pachyderma, Globorotalia inflata. Cette association est plutôt caractéristique d'une province tempérée froide [3]. Dans certaines carottes (KS05, KS07), on observe quelques spécimens de Neogloboquadrina humerosa, espèce dont l'extinction est datée au Pleistocène basal [47]. Globorotalia truncatulinoides, marqueur du Quaternaire, et Orbulina universa, forme d'eau tempérée [3] sont très rares. Globalement, les associations observées correspondent à un faciès de plate-forme, excluant cependant la zone médiolittorale, dans un contexte bathymétrique inférieur ou égal à $100 \mathrm{~m}[2,4]$.

Ces données impliquent: (1) soit que l'association de foraminifères s'est déposée in situ lors d'un bas niveau marin relatif ; (2) soit que le sédiment qui les contient provient des zones côtières. Dans ce cas, il peut également être ancien, mais l'inversion observée entre les formes planctoniques et benthiques n'est alors pas caractéristique d'un bas niveau marin au moment du dépôt. À l'exception de quelques systèmes émergés sur les côtes anglaises [20] ou de petits systèmes côtiers bretons [46], très peu de formations d'âge Pliocène ou Pléistocène basal sont observées dans le domaine côtier de la Manche, de sorte que la première hypothèse est la plus vraisemblable. Cependant, les données fournies par les autres groupes indiquent que, si ce sédiment est autochtone (issu du même intervalle bathymétrique), il a été remanié.

\section{DISCUSSION}

\subsection{Dynamique sédimentaire}

Les observations confirment le caractère très évolué des sédiments de la plate-forme distale, en accord avec le modèle de Wilson $[59,60]$. Cette maturité est marquée par la diversification des assemblages fauniques autochtones, avec notamment d'importantes populations de bryozoaires et de serpules. En outre, les associations mises en évidence comportent des populations exotiques : une population moderne essentiellement allochtone et une population ancienne autochtone. Les échantillons ne contiennent aucune thanatofaune moderne qui serait exclusivement typique de la plate-forme profonde (pas de mytilus à couleur préservée). En revanche, une thanatofaune blanche de formes côtières très fraîches prédomine (échinides, gastéropodes, bivalves, ostracodes et cirripèdes). À côté de cette faune moderne, on note la présence de formes arctico-boréales de couleur jaunâtre à fauve (bivalves, cirripèdes, foraminifères), d'âge Weichsélien probable, ainsi que celle, plus rare, d'espèces d'âge Pliocène ou Pleistocène inférieur, en particulier des foraminifères et des bivalves (ces derniers actuellement restreints au domaine méditerranéen). Ce mélange de sources d'âges différents met en évidence une homogénéisation mécanique du sédiment.

\subsubsection{Partitionnement vertical des associations : effet des tempêtes}

Le contenu thanatofaunique ne présente aucune évolution stratigraphique et des espèces typiques de biotopes très différents sont présentes dans les mêmes niveaux. La 
continuité (ou la discontinuité) de la présence d'une espèce le long d'une carotte reflète principalement sa proportion dans le sédiment au-dessus (ou en-dessous) d'une valeur seuil qui est celle de sa détection dans le volume échantillonné. Chez les bivalves, parmi les espèces les plus abondantes, certaines sont ubiquistes dans les carottes (Chlamys opercularis), tandis que d'autres n'apparaissent massivement que dans une seule carotte (effet de banc ; ex. Glycymeris glycymeris dans la carotte $\mathrm{KS} 08$; figure 2). En revanchc, dans chaque carottc, certains niveaux présentent une concentration plus élevée en bivalves de divers groupes (ex. KS05, 20-40 et 120-140 $\mathrm{cm}$; figure 2). Les niveaux dits riches en bivalves (entre 5 et 12 taxons par échantillon) alternent avec des niveaux dits pauvres (de 0 à 4 taxons) qui se correspondent d'une carotte à l'autre sur les premiers $80 \mathrm{~cm}$ de sédiment. Au-delà, l'alternance est moins nette et plus aléatoire, principalement à cause du nombre réduit de carottes à comparer.

Le partitionnement vertical de la richesse en taxons des carottes est interprété comme un effet de ségrégation des coquilles lors des épisodes de remaniement par les courants, notamment lors d'épisodes de tempêtes. Les individus de petite taille et de mobilité maximale sont concentrés dans les niveaux à matrice plus fine (ex. KS06, 20-40 cm : ostracodes ; 180-200 cm : petits oursins tels que Echinocyamus pusillus ; figure 2), ou resédimentés immédiatement au-dessus des lits de graviers témoins de la remobilisation (ex. KS08, 80-100 cm ; figure 2). Ce tri hydrodynamique sélectif des petites particules conduit à un appauvrissement taxonomique, avec notamment la disparition des bivalves dans les passées de sable fin (ex. KS06, 0-20 cm ; figure 2). L'homogénéisation par la houle du sédiment superficiel serait sporadique et brève, dans la mesure où des formes actuelles fragiles (échinides ou bivalves juvéniles) sont présentes à la base comme au sommet des carottes.

\subsubsection{Importation d'espèces modernes: rôle des courants résiduels}

Les thanatofaunes modernes d'origine allochtone prédominent. L'importation est caractérisée par des assemblages frais typiques du domaine infralittoral dans les populations d'ostracodes, de gastéropodes et d'oursins. La présence d'une thanatofaune infralittorale fraîche dans les sédiments étudiés reflète le transport résiduel vers l'ouest-sud-ouest dû à la prédominance du jusant sur toute la plate-forme des approches occidentales de la
Manche. Alors que les pics de courant sont plutôt NNESSW dans la zone d'étude, cette direction de transport pourrait s'expliquer par un effet d'asymétrie de l'ellipse tidale [51].

À cet effet pourrait s'ajouter, dans les zones sources infralittorales du sud-ouest de la Bretagne, l'influence de la dérive littorale sud-armoricaine [50]. De fortes composantes de mouvement résiduel des masses d'eau près du fond ont été mises en évidence dans la zone d'étude par Cavanié et Hyacinthe [15] (figure 1: point 2), Pingree et Le Cann [50], ainsi qu'au point Krogen [51] (figure 1: point 1). À long terme, l'action combinée des courants résiduels, dont la vitesse moyenne sur un cycle tidal ne dépasse pas quelques centimètres par seconde [50], justifierait la présence des associations infralittorales sur la plate-forme profonde (figure 3).

\subsubsection{Variabilité dans l'espace de la richesse taxonomique : rôle de la circulation tidale?}

La variation d'une carotte à l'autre de la richesse taxonomique (ou richesse spécifique, dans le cas de taxons identifiés au niveau de l'espèce) pourrait être un traceur potentiel des trajectoires sédimentaires dans la zone du banc (figure 3). À cause du faible nombre de carottes comparées, on ne peut écarter la participation à cette variabilité des facteurs locaux (pièges topographiques, par exemple). Cependant, les carottes prélevées sur le flanc est du banc (KS03 et KS05) montrent une plus grande richesse taxonomique relative que les carottes prélevées sur le flanc ouest (KS06 et $\mathrm{KS07}$; figure 3). Les carottes KS04 et KS08, prélevées au sommet du banc, présentent des caractéristiques intermédiaires (figure 3).

Cette répartition de la richesse taxonomique dans les sédiments superficiels du banc suggère un transport sédimentaire contrôlé par la dynamique tidale, le sédiment importé sur le flanc est du banc étant transporté vers le sud sous l'effet du courant de jusant, puis repris en partie sur le flanc ouest du banc où il est transporté vers le nord sous l'effet du courant de flot (figure 3). Au cours de ce transport, les associations de thanatofaunes s'appauvriraient en réponse au tri hydrodynamique des bioclastes selon leur taille, leur forme et leur densité. Les fractions incorporées au système sur le flanc ouest seraient globalement plus pauvres en thanatocénoses littorales que celles incorporées sur le flanc est, parce qu'elles correspondant à un sédiment importé antérieurement sur la plate-forme et donc trié depuis plus longtemps. 


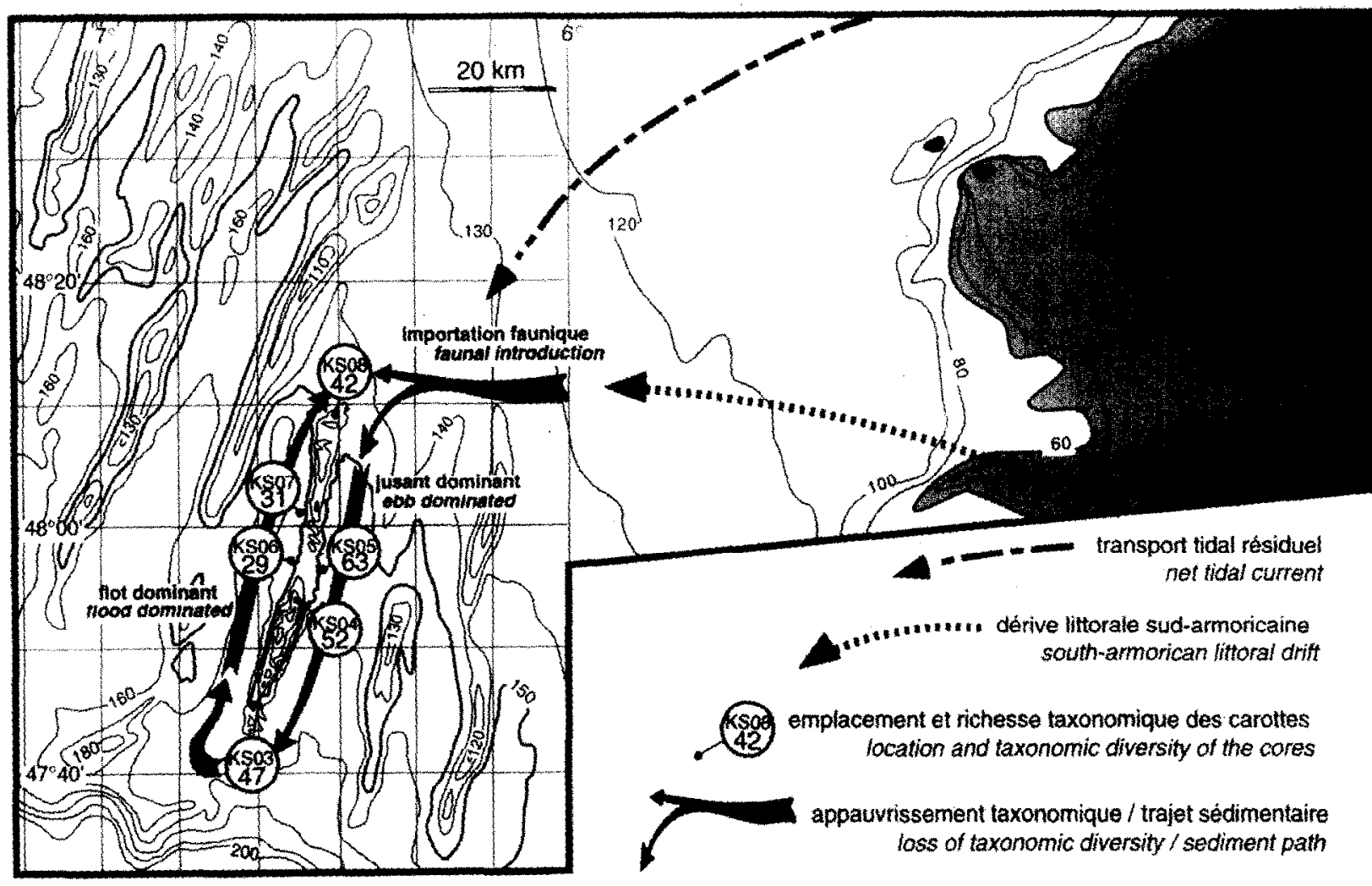

Figure 3. Relation schématique entre courants résiduels et transports sédimentaires sur le banc du Kaiser. Pour chaque carotte (KS03 à KS08), le numéro mentionné dans la pastille correspondante représente la richesse taxonomique. La richesse taxonomique est comprise comme le nombre de segments de $20 \mathrm{~cm}$ présentant, dans l'intervalle $0-80 \mathrm{~cm}$ (commun à toutes les carottes), un ou plusieurs taxons se rapportant aux groupes rapportés sur la figure 2. Carte bathymétrique d'ensemble d'après Bouysse et al. [9]. Carte bathymétrique détaillée du banc du Kaiser d'après Reynaud [51].

Figure 3. Schematic relationship between residual currents and net transport around the Kaiser bank. For each core (KS03 to KS08), the number in the corresponding round spot represents the taxonomic diversity (i.e. the number of segments of $20 \mathrm{~cm}$ displaying one or several taxons as mentioned in figure 2 within the depth interval $0-80 \mathrm{~cm}$, which is common to all cores). Bathymetric map of the banks after Bouysse et al. [9]. Detailed bathymetric map of the Kaiser bank after Reynaud [51].

Malgré le nombre restreint de carottes, cette interprétation vérifie le schéma dynamique développé pour le banc à partir de l'observation des corps sédimentaires tidaux actifs présents à la surface du banc [51]. L'inversion du transport résiduel d'un flanc à l'autre est une caractéristique des banes tidaux actifs $[31,33]$, la rotation du sédiment à l'extrémité des bancs ayant été directement observée sur certains édifices actifs du sud de la mer du Nord $[45,32]$. Le schéma de transport suggéré par l'analyse des thanatofaunes implique l'absence de transferts sćdimentaires significatifs à travers la crête du banc (figure 3). Cette caractéristique, contraire au modèle dynamique des bancs tidaux [33], est cependant confirmée par l'étude des dunes tidales présentes au sommet du banc [51].

\subsubsection{Remaniements d'espèces anté-holocènes: rôle des variations du niveau marin}

Le remaniement de formations anciennes dans le sédiment superficiel est suggéré par certaines formes de bivalves, cirripèdes et ostracodes. En effet, les formes arctico-boréales rencontrées ne vivent actuellement pas en-dessous de la latitude d'Édimbourg (limite nord de la province climatique de la mer Celtique d'après Hall [26]) mais existaient en mer Celtique et en Manche lors de la dernière glaciation. Or, les transports sédimentaires résiduels en mer d'Écosse et en mer du Nord ne portent pas aujourd'hui vers le sud [34]. Par ailleurs, la distance qui sépare ce biotope arctico-boréal du banc du Kaiser semble trop importante pour que les thanatofaunes froides 


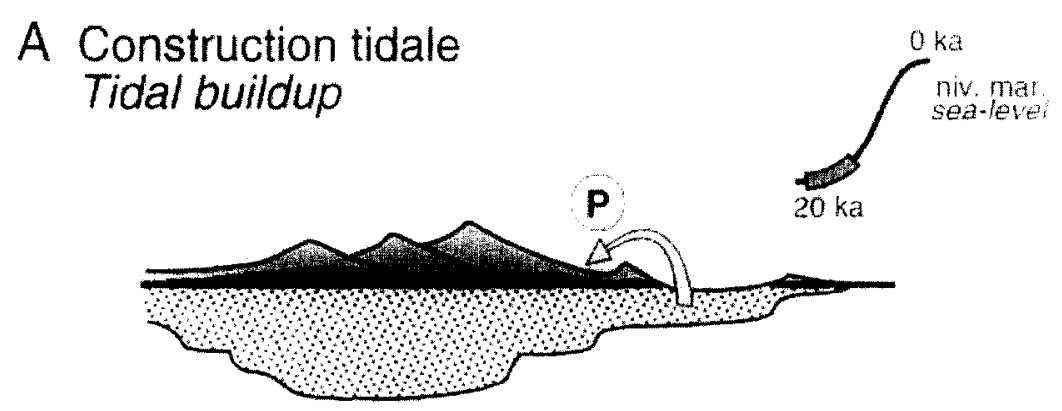

B Scellement (débâcle glaciaire) Sealing (glacial outwash)

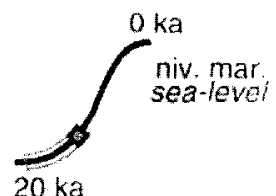

$20 \mathrm{ka}$

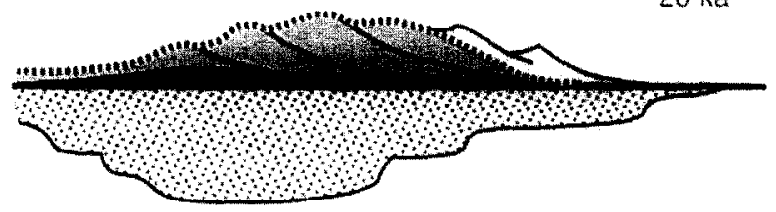

\section{Remaniement profond (chenaux tidaux ?) Deep reworking (tidal channels ?)}

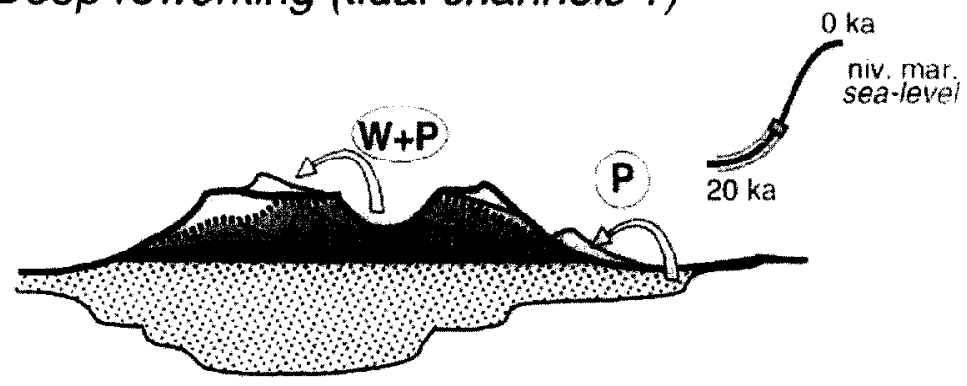

\section{Remaniement superficiel (tempêtes) Surficial reworking (storms)}

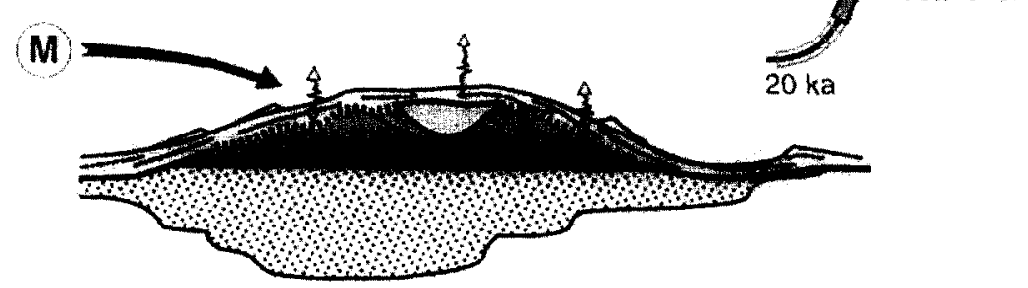

Figure 4. Hypothèse de formation du sédiment superficiel de la plate-forme profonde des approches occidentales de la Manche lors de la dernière transgression glacio-eustalique A : formation des bancs celtiques par accumulation d'un épandange biolithoclastique weichsélien ; $B$ : formation de la semelle graveleuse du sédiment superficiel au moment de la débâcle glaciaire. $\mathrm{C}$ : incorporation dans le stock superficiel des produits de remaniement des bancs et des vallées pliocènes sousjacentes (à la faveur de la résonance tidale dı bassin des approches occidentales de la Manche ?) ; D : importation de thanatofaunes infralittorales après l'installation des courants modernes de dérive vers l'ouest (voir figure 3). Trame de points : remplissage pliocène de vallée incisée. Gris foncé : Quaternaire non remanié. Gris clair: banc formé au début de la transgression flandrienne. Blanc: sédiment superficiel. Tireté gras : semelle graveleuse du sédiment superficiel holocène (représenté en blanc). Traits-points: discontinuités internes du sédiment superficiel liées à des remanicments modernes par les tempêtes. $\mathbf{P}$ : faunes d'âge Pliocène à Pléistocène basal. W : faunes weichséliennes (essentiellement 20-14 Ka). $\mathrm{M}$ : thanatofaunes actuelles importées.

Figure 4. Hypothesis for the formation of the surficial sediments of the deep outer shelf in the Western Channel Approaches during the last post-glacial sea-level rise. A: build-up of the Celtic Banks on a Weichselian glacial biolithoclastic sandy substrate; $B$ : formation of the basal lag of the surficial sediment blanket by glacial outwash over the sand banks. $\mathrm{C}$ : addition of Weichselian and Pliocene faunas in the surficial mobile sediment by reworking of the sand banks and carving of the incised valleys by strong tidal currents during a resonance stage of the transgressive celtic basin; D: incoming of coastal faunas after installment of the western sediment drift (see figure 8). Dotted screen: Pliocene incised valley fill. Dark shadow: non-reworked older Quaternary. Medium shadow: late Weichselian sand bank. White: surficial sediment. Bold dotted line: gravel lag at the base of the surficial sediment blanket (shown in white, with intra non-conformities representing storm reworking). P: Pliocene-lowermost Pleistocene faunas. W: Weichselian faunas (merely 20-14 Ka). M: supplied modern faunas. 
identifiées, relativement bien conservées, en soient issues. Il est donc vraisemblable que ces faunes soient autochtones et datent de la glaciation wechsélienne. Leur ancienneté est accréditée par leur couleur jaunâtre à fauve et leur patine caractéristiques des sables bioclastiques marins glaciaires de mer Celtique méridionale $[7,37,30]$.

En revanche, on ne retrouve pas dans la thanatofaune « héritée » (de couleur fauve) les espèces « chaudes» comme le bivalve Eastonia rugosa et le gastéropode Astraea rugosa, présents en Manche à l'Éemien, vers $125000 \mathrm{BP}$ [40]. Ces espèces, absentes actuellement sur la façade atlantique, au nord de la zone lusitanienne, se trouvaient cependant en mer Celtique à l'Éemien. Cependant, le sédiment contient des spécimens de bivalves et de foraminifères planctoniques d'âge antérieur au Pleistocène basal. Il est à noter que le secteur d'étude est demeuré immergé durant tout le Quaternaire, comme le suggèrent les modèles isostatiques les plus récents [36]. En conséquence, l'héritage faunique des sédiments superficiels du Kaiser implique (1) des remaniements successifs au cours du Weichsélien, dont résulterait la disparition des formes éemiennes soit par transfert dans la pente continentale sous l'effet du jusant résiduel [34], soit par frottement grain sur grain [60], et (2) l'introduction, plus récemment dans le Weichsélien, des formes d'âge Pliocène-Pléistocène basal.

\subsection{Formation du sédiment}

\subsubsection{Sources anciennes du sédiment}

Si l'absence de faune quaternaire pré-weichsélienne résulte d'un phénomène d'usure liée au remaniement fréquent par les agents hydrodynamiques, la fraction d'âge Pliocène-Pléistocène basal (foraminifères et bivalves) a dû être introduite lors du dernier cycle climatique dans le sédiment superficiel. Cette importation a pu se produire lors de remaniements au cours du dernier bas niveau marin glacio-eustatique et/ou des premiers stades de la transgression suivante. D'après la littérature, deux formations meubles pourraient être pré-weichséliennes: les bancs celtiques et les vallées incisées de la plate-forme profonde.

L'hypothèse que les bancs celtiques sont des reliques de prismes de bas niveau marin plus anciens que le dernier maximum glaciaire [51,5] suggère que les bancs correspondent aux reliques d'un système deltaïque ou estuarien mis en place à l'aval du « Fleuve Manche» lors des bas niveaux marins glacio-eustatiques du Quaternaire supé- rieur [22]. Alternativement, les bancs résulteraient du remaniement total de ces dépôts sous l'action de courants tidaux à la faveur de la dernière transgression postglaciaire $[9,48,52,61]$. Cette deuxième interprétation semble la plus probable, dans la mesure où elle seule justifie l'absence de faunes Quaternaires pré-weichséliennes dans les produits de remaniement : un remaniement total à chaque bas niveau marin est nécessaire pour expliquer le rajeunissement du stock bioclastique et l'abrasion des spécimens hérités du cycle précédent. Les spécimens d'âge Pliocène à Pléistocène basal n'ont alors pas les bancs pour formation source.

La fraction d'âge anté-Pléistocène inférieur du sédiment proviendrait du remaniement des dépôts comblant la quasi-totalité des vallées incisées de la mer Celtique. Ces dépôts sont attribués au Pliocène supérieur ou au Pléistocène basal [21]. L'architecture du comblement suggère une mise en place polyséquentielle évoluant depuis le domaine fluviatile à la base vers un milieu estuarien au sommet [51]. Cela rend plausible l'origine dans ce milieu des foraminiferres plus côtiers observés dans le sédiment superficiel. En outre, l'hypothèse d'une meilleure préservation du remplissage des vallées que des bancs se justifie par la position «protégée » des vallées sous le niveau de base du plateau continental adjacent, de sorte que leur excavation par des courants aurait été plus difficile que l'aplanissement des bancs par la houle. Ainsi, seule une petite partie du matériel contenu dans les paléovallées aurait été réintroduite dans le sédiment superficiel à chaque bas niveau marin, de sorte que leur remplissage est peu érodé $[9,21]$.

\subsection{2. Âge et mode de mise en place}

L'interprétation ci-dessus permet de proposer un scénario de mise en place des formations superficielles de la mer Celtique postéricurement au dernicr bas niveau marin glacio-eustatique (figure 4). Les bancs se construiraient par 40-60 m de fond en domaine de plate-forme tidale [52], et remanieraient en partie le remplissage des vallées incisées sous-jacentes. D'après les modèles récents de variation du niveau marin relatif, cet épisode interviendrait au début de la dernière transgression glacio-eustatique, entre $20000 \mathrm{BP}$ et $15000 \mathrm{BP}$ [36], voire vers $11000 \mathrm{BP}$ dans l'hypothèse d'un fort contrôle isostatique [61]. Les bancs constitueraient alors un stock marin dans lequel serait piégée temporairement, outre les faunes weichséliennes synchrones de leur croissance, une petite partie du matériel Pliocène à Pléistocène basal issu 
des vallées sous-jacentes (figure 4a). La débâcle tardiglaciaire serait à l'origine de la semelle graveleuse du sédiment superficiel, qui scellerait le matériel plio-quaternaire ( « layer B » de Pantin et Evans [48] ; figure 4b). Cette couche de scellement serait postérieurement percée sous l'effet d'un remaniement profond des bancs, dont témoigne un ensemble de chenaux comblés, peut-être d'origine tidale $[52,57]$. À la faveur de ces incisions, une fraction du matériel composite plio-quaternaire serait réintroduite dans les niveaux superficiels (figure $4 c$ ).

Avec la progression de la transgression holocène, une forte autoproduction bioclastique du système de dépôt se développerait, en réponse principalement à la diminution de l'intensité des courants tidaux [52]. Cependant la dynamique demeurerait intense et les espèces produites in situ (circalittorales) donneraient une malacofaune triée sur sa résistance à l'abrasion, comme en témoigne par exemple l'oligospécificité des populations de bivalves observées. Le système s'enrichirait progressivement d'une fraction infralittorale transportée sur le fond depuis les zones côtières, à la faveur d'une prédominance du jusant dans les approches occidentales de la Manche (figures 3 et $4 d$ ). L'homogénéisation de la couverture superficielle se ferait à la faveur (1) de « l'autocannibalisation » du système par les dunes tidales modernes et (2) des remobilisations liées aux tempêtes (figure $4 d$ ). Ces dernières produiraient un tri sélectif des individus, qui se retrouvent concentrés selon leur forme, leur taille et leur densité dans des passées correspondant à des dépôts de vannage (gros bivalves associés à des lits de graviers) ou de décantation (radioles d'oursins, petits gastéropodes, ostracodes, associés à un sédiment fin).

\section{CONCLUSION}

L'étude des associations thanatofauniques dans le sédiment superficiel du banc du Kaiser (approches occidenta- les de la Manche) permet de préciser les hypothèses concernant la dynamique et les sources du sédiment.

- La répartition des taxons, dans les carottes et d'une carotte à l'autre, éclaire les connaissances antérieures de la dynamique actuelle du sédiment superficiel. La ségrégation des particules dans des passées décimétriques selon leur forme, leur taille ou leur densité traduit un remaniement sporadique par les tempêtes. L'analyse préliminaire de la variation de la richesse taxonomique relative d'une carotte à l'autre suggère un schéma de transport du sédiment autour du banc qui corrobore les résultats fournis par l'analyse des corps sédimentaires actifs à la surface du banc.

- Plusieurs thanatocénoses très différentes sont mélangées dans les associations fauniques. Outre la source autochtone moderne correspondant au domaine circalittoral du banc, on distingue: (1) une source allochtone moderne, constituée par une association infralittorale typique des côtes bretonnes ; (2) une source autochtone ou pseudo-autochtone ancienne, comprenant une association d'âge Pliocène à Pléistocène basal et une association d'âge Weichsélien. Ces deux associations pourraient être issues respectivement des vallées incisées et des bancs de la mer Celtique, remaniés lors des derniers épisodes glaciaires et au début de la dernière transgression glacioeustatique.

\section{Remerciements}

Les carottes Sédimanche 2 ont été prélevées dans le cadre du programme MAST2-CT92-0029. La carte bathymétrique du banc du Kaiser a été obtenue à partir de données multifaisceaux traitées par A. Normand (Ifremer). Merci à R. Kerbrat et G. Floch (Ifremer) pour leur participation au travail sur les carottes, ainsi qu'à A. El Hakour (université de Nice) et C. Perrin (Muséum national d'histoire naturelle) pour leur participation aux déterminations fauniques.

\section{RÉFÉRENCES}

[1] Auffret G.A., Dynamique sédimentaire de la marge continentale Celtique : évolution Cénozoique - spécificité du Pléistocène supérieur et de l'Holocène, doct. état, univ. Bordeaux-I, $1983,335 \mathrm{p}$.

[2] Bé A.W.H., Ecology of Recent planktonic foraminifera: Part 2 - Bathymetric and seasonal distributions in the Sargasso Sea off Bermuda, Micropal. 6 (1960) 373-392.
[3] Bé A.W.H., An ecological, zoogcographic and taxonomic review of recent planktonic foraminifera, in: Ramsay A.T.S. (Ed.) Oceanic Micropal. 1, 1977, pp. 1-100.

[4] Berger W.H., Ecologic patterns of living planktonic Formanifera, Deep-Sea Res. 16 (1969) 1-24.

[5] Berné S., Marsset T., Lericolais G., Bourillet J.-F., De Batist M., Erosional offshore sand ridges and lowstand shorefaces: 
examples from tide and wave dominated environments around France, J. Sed. Res. 68 (1998) 540-555.

[6] Bouchet P., Danrigal F., Huygens C., Coquillages des côtes atlantiques et de la Manche, Ed. Pacifique, Papeete, 1978, 145 p.

[7] Bourcart J., Les sables roux du Plateau continental français, C. R. Acad. Sci. Paris 225 (1947) 316-318.

[8] Bourillet J.-F., Loubrieu B., Carte bathymorphologique de la marge des cntrécs de la Manche (1:250000 à $46^{\circ}$ Nord), Ifremer, 1995.

[9] Bouysse P., Horn R., Lapierre F., Lelann F., Étude des grands bancs de sable du Sud-Est de la mer Celtique, Mar. Geol. 20 (1976) 251-275.

[10] Bouysse P., Lelann F., Scolari G., Les sédiments superficiels des approches occidentales de la Manche, Mar. Geol. 29 (1979) 107-135.

[11] Buchanan J.B., The biology of Echinocardium cordatum (Echinodcrmata: Spatangoidea) from different habitats, J. Mar. Biol. Ass. U.K. 46 (1966) 97-114.

[12] Carruthers J. N., History, sand waves and near-bed currents of La Chapelle Bank, Nature 197 (1963) 942-946.

[13] Cartwright D., Stride A.H., Large sand waves near the edge of the continental shelf, Nature 181 (1958) 41.

[14] Cavanié A., Application à la zone au large du Finistère d'une méthode d'estimation statistique des courants de houle au voisinage du fond, La Houille Blanche 7 (1975) 497-500.

[15] Cavanié A., Hyacinthe J.L., Étude des courants et de la marée à la limite du Plateau continental d'après les mesures effectuées pendant la campagne "golfe de Gascogne 1970 ", CNEXO, 1976.

[16] Channon R.D., Hamilton D., Wave and tidal current sorting of shelf sediments, southwest of England, Sedimentology 23 (1976) 17-42.

[17] Cornet M., Recherches sur l'écologie des mollusques bivalves du Plateau continental Sud-Gascogne, Ann. Inst. Océanogr. 61 (1985) 59-74

[18] Darwin C., A monograph on the subclass Cirripedia, with figures of all species, the Balanidae, the Verrucidae, etc., Roy. Soc. London, 1854, $684 \mathrm{p}$.

[19] David B., Laurin B., De Ridder C., How Echinocardium cordatum (Pennant) shows sexual dimorphism, in: Burke R.D. (Ed.), Echinoderm Biology, Balkema, Rotterdam, 1988, pp. 683-686.

[20] Evans C.D.R., The geology of the western English Channel and its western approaches, HMSO, London, 1990, $93 \mathrm{pp}$.

[21] Evans C.D.R., Hughes M.J., The Neogene Succession of the South Western Approaches, Great Britain, J. Geol. Soc. London 141 (1984) 315-326.

[22] Gibbard P.L., The history of the great northwest European rivers during the past three million years, Phil. Trans. Roy. Soc. London B 318 (1988) 559-602.

[23] Glémarec M., Distribution bathymétrique et latitudinale des Bivalves du golfe de Gascogne, Haliotis 9 (1978) 23-32.
[24] Glémarec M., Les facteurs déterminant la distribution des bivalves actuels sur les plates-formes Bord-Est atlantiques, Géol. Médit. 15 (1988) 73-81.

[25] Guillaume M.-C., Peypouquet J.-P., 'letard J., Quaternaire et actuel, in: Oertli M. (Ed.), Atlas des ostracodes de France, Bull. Centr. Rech. Explor.-Prod. Elf Aquitaine 9 (1985) 337-377.

[26] Hall C.A., Shallow water marine climates and molluscan provinces, Ecology 4 (1964) 226-233.

[27] Hamilton D., Sommerville J.H., Stanford P.N., Bottom currents and shelf sediments, Southwest of Britain, Sediment. Geol. 26 (1980) 115-138.

[28] Heathershaw A.D., New A.L., Edwards P.D., Internal tides and sediment transport at the shelf break in the Celtic Sea, Cont. Shelf Res. 7 (1987) 485-517.

[29] Heezen B.C., Hollister C.D., Deep-sea current evidence from abyssal sediments, Mar. Geol. 1 (1964) 141-174.

[30] Hinschberger F., L'Iroise et les abords d'Ouessant et de Sein, étude de morphologie et de sédimentologie sous-marines, doct. état, universités Paris-Caen, 1970.

[31] Houbolt J.J.H.C., Recent sediments in the southern bight of the North Sea, Geol. en Mijnb. 47 (1968) 245-273.

[32] Howarth M.J., Huthnance J.M., 'lidal residual currents around a Norfolk sand bank, Est. Coast. Shelf Sci. 19 (1984) 105-117.

[33] Kenyon N.H., Belderson R.H., Stride A.H., Johnson M.A., Offshore tidal sand-bank as indicator of net sand transport and as potential deposits, Spec. Publs. Int. Ass. Sedim. 5 (1981) 257-268.

[34] Kenyon N.H., Stride A.H., The tide-swept continental shelf sediments between the Shetland Isles and France, Sedimentology 14 (1970) 159-173.

[35] Koehler R., Faune de France, Lechevallier, Paris, 1921, 210 p.

[36] Lambeck K., Late Devensian and Holocene shorelines of the British Isles and North Sea from models of glacio-hydro-isostatic rebound, J. Geol. Soc. London 152 (1995) 437-448.

[37] Lapierre F., Étude de la répartition des sédiments dans le golfe de Gascogne, Bull. Inst. Géol. Bassin Aquitaine 3 (1967) 93126.

[38] Larsonneur C., Bouysse P., Auffret J.-P., The superficial sediments of the English Channel and its Western Approaches, Sedimentology 29 (1982) 851-864.

[39] Lauriat-Rage A., Les bivalves du Pliocène de Normandie, Bull. Mus. Nat. Hist. Nat. ${ }^{e}$ sér. 8 C (1986) 3-51.

[40] Lauriat-Rage A., Paleontological data about the climatic trends from Chattian to present along the Northeastern Atlantic frontage, in: First Congress R.C.A.N.S. Ciencias de la terra, UNL, Lisboa, 1993, pp. 167-169.

[41] Laurin B., Marchand D., Thierry J., Variations morphologiques du test chez Echinocardium cordatum (Pennant) : étude qualitative et quantitative de cinq échantillons de Bretagne et Normandie, Bull. Soc. Géol. Norm. et Amis du Muséum du Havre 65 (1979) 89-104.

[42] Lericolais G., Évolution Plio-Quaternaire du Fleuve Manche : stratigraphie et géomorphologie d'une plate-forme continen- 
tale en régime Périglaciaire, doct. univ. Bordeaux-I, 1997. $265 \mathrm{p}$.

[43] Lericolais G., Guennoc P., Auffret J.-P., Bourillet, J.-F. Berné S., Detailed survey of the western end of the Hurd Deep (English Channel): New facts for a tectonic origin, in: De Batist M., Jacobs P. (Ed.), Geology of Siliciclastic Shelf Seas. Spec. Publ. Geol. Soc. London 117 (1996) 203-217.

[44] McCave I.N., Transport et escape of fine grained sediment from shelf areas, in: Swift D.J.P., Duane D.B., Pilkey O.H. (Ed.), Shelf sediment transport, Dowden-Hutchinson and Ross Inc., Stroutdsburgh, Pennsylvania, 1972, pp. 225-248

[45] McCave I.N., Langhorne D.N., Sand waves and sediment transport around the end of a tidal sand bank, Sedimentology 29 (1982) 95-110.

[46] Morzadec-Kerfourn M.-T., De l'importance relative des transgressions et régressions marines glacio-eustatiques sur le Massif armoricain, Rev. archéol. Ouest, suppl. n ${ }^{\circ} 2$ (1990) 37-40.

[47] Moullade M., Upper Neogene and Quaternary planktonic foraminifers from the Blake Outer Ridge and Blake Bahama Basin (Western North Atlantic), Deep Sea Drilling Project Leg 76 , Sites 533 and 534, in: Sheridan R.E., Gradstein F.M. (Ed.), Init. Repts. DSDP 76, Washington (U.S. Govt. Print. Office), 1983, pp. 511-535.

[48] Pantin H.M., Evans C.D.R., The Quaternary History of the Central and Southwestern Celtic Sea, Mar. Geol. 57 (1984) 259-293.

[49] Péres J.M., Picard J, Nouveau manuel de bionomie benthique de la mer Méditerranée, Rec. Trav. Stat. mar. Endoume 31 (1964) 1-137.

[50] Pingree R.D., Le Cann B., Celtic and Armorican slope and shelf residual currents, Prog. Oceanogr. 23 (1989) 303-338.

[51] Reynaud J.-Y., Architecture et évolution d'un banc sableux de mer Celtique méridionale, doct. univ. Lille, 1996, 185 p.
152] Reynaud J.-Y., Tessier B.. Proust J.-N., Dalrymple R.W. Marsset T., De Batist M. Bourillet J.-F., Lericolais G., Eustatic and hydrodynamic controls on the architecture of a deep shelf sand bank (Celtic Sea), Sedimentology (sous presse).

[53] Sleath J.F.A.. Velocities and shear stresses in wave-current flows, J. Geophys. Res. 96 (1991) 15237-15244.

[54] Southward A.J., Crisp D.J., Les cirripèdes des mers européennes, in: Catalogue des principales salissures marines (rencontrées sur les coques de navires dans les eaux européennes) I : Balanes, Organisation de coopération et de développement économiques. 1963. pp. 9-46.

[55] Stride A.H., Current-swept sea-floors near the the southem half of Great Britain, Quat. J. Geol. Soc. London 119 (1963) 175-199.

[56] Tebble N., British Bivalve seashelf, The British Museum (Natural History), London, 1966, 212p

[57] Tessier B., Expressions sédimentaires de la dynamique tidale. Mémoire d'habilitation à diriger des recherches, Lille I, 1997. $79 \mathrm{p}$.

[58] Tortonese E., Fauna d'Italia VI: Echinodermata. Calderini, Bologna, 1965, $419 \mathrm{p}$.

[59] Wilson J.B., Shelly faunas associated with temperate offshore tidal deposits, in: Stride A.H. (Ed.), Offshore tidal sands: processes and deposits, Chapman and Hall, Paris, 1982, pp. 126-172.

[60] Wilson J.B., A model for temporal changes in the faunal composition of shell gravels during a transgression on the continental shelf around the British Isles, Sediment. Geol. 60 (1988) 95-105.

[61] Wingfield R., A model of sea-levels in the Irish and Celtic seas during the end-Pleistocene to Holocene transition, Geol. Soc. Spec. Publ. 96 (1995) 209-243.

[62] Wood S.V., A monograph of the Crag Mollusca, Part II: Bivalves. Paleontological Society, London, 1851-1882, 504 p. 